Military Technical College, Kobry El-Kobbah, Cairo, Egypt

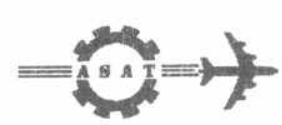

$9^{\text {th }}$ International Conference

On Aerospace Sciences \& Aviation Technology

\title{
IMPROVED LOCALLY DISTORTED CONFORMAL FDTD ALGORITHM FOR MODELING 3D STRUCTURE
}

\author{
MITKEES A A. A., ALLAM A. M., ALI*^ O. M. N
}

\section{ABSTRACT}

This paper, presents an improved algorithm for the conformal finite-difference timedomain. Locally distorted conformal FDTD griding methods are based on a regular orthogonal grid, with the exception that grid is deformed in the near vicinity of boundary interface. It is based on the concept of weighted-volume effective dielectric constant. It models an arbitrarily shaped dielectric objects. Modeling the cylindrical dielectric resonator shows the accuracy and usefulness of the method.

\section{KEY WORDE}

Finite-Difference Time-Domain (FDTD) method, Dielectric resonator.

\footnotetext{
* Egyptian Armed Forces.

** Syrian Armed Forces.
} 


\section{INTRODUCTION}

The finite-difference time-domain (FDTD) method [1-4] has been proven to be a very efficient numerical algorithm in computational electromagnetics. The traditional FDTD algorithm is based on a Cartesian coordinate system, and is difficult to exactly generate meshes for electromagnetic structures with curved boundaries. It is usual to utilize a staircase approximation into a FDTD method for curved structures and an accurate solution can only be obtained using very fine grids and consequently, a very small time step.

Apart from the staircase approximation, there are three different commonly used types of the FDTD method, which have been put forward to analyze electromagnetic structures with curved boundaries: The conformal or contour-path algorithm [5], formulas in the curvilinear coordinate system [6], and nonorthogonal FDTD method [7]

Reference 8, has reported a simple yet accurate technique for the FDTD analysis of curved dielectric bodies using a locally conformal grid. This approach requires neither a borrowing nor interpolating of the electric fields at the object boundary. This paper calculates the effective dielectric constant at the boundary by taken the weighted volume average of the two dielectrics, and then calculate the electric and magnetic field from FDTD equations, these equations have been used without approximation as in reference [8]. The resonance frequencies of the cylindrical dielectric resonator show the accuracy of this method with respect to the conformal finite-difference timedomain [8] and staircase FDTD grid

\section{ALGORITHM}

One begins by the conformal finite-difference time-domain (CFDTD) method [8], it presents the following representative equation for the electric field along the $x$ direction:

$$
\left.E_{x}\right|_{i, j, k} ^{n+1}=\left.E_{x}\right|_{i, j, k} ^{n}+\frac{\Delta t}{\varepsilon} \cdot\left\{\frac{\left.H_{z}\right|_{i, j+1, k} ^{n+1 / 1}-\left.H_{z}\right|_{l, j, k} ^{n+1 / k}}{\Delta y} \frac{\left.H_{y}\right|_{i, j, k+1} ^{n+1 / 2}-\left.H_{y}\right|_{i, j, k} ^{n+1 / k}}{\Delta z}\right\}
$$

where $\Delta y$ and $\Delta z$ are the step sizes along the $y$-and z-direction respectively. Similar equations can be written for other electric-field components. The equation for the $z$ direction magnetic field is:

$$
\begin{array}{r}
\left.\left.H_{z}\right|_{i, j, k} ^{n+1 / z=H_{z}}\right|_{i, j, k} ^{n-1 / k}+\frac{\Delta t}{\mu A_{z}(i, j, k)} \cdot\left\{\left.E_{x}\right|_{t, j, k} ^{n} \cdot l_{x}(i, j, k)-\left.E_{x}\right|_{i, j-i, k} ^{n} \cdot l_{x}(i, j-j, k)\right. \\
\left.-\left.E_{y}\right|_{i, j, k} ^{n} \cdot l_{y}(i, j, k)+\left.E_{y}\right|_{i, 1, j, k} ^{n} \cdot l_{y}(i-1, j, k)\right\}
\end{array}
$$

where $A_{z}$ is the face area of the cell, excluding the dielectric region, $l_{x}$ and $l_{y}$ are the cell lengths outside the metal, along the $x$-and $y$-directions, respectively. 


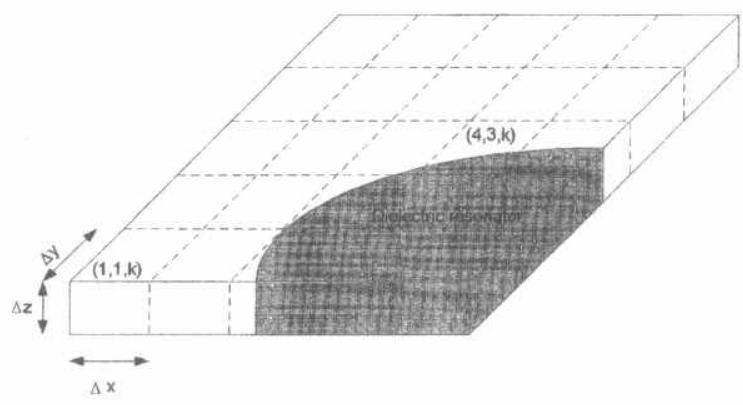

Fig. 1 One-quarter cross section of a cylindrical DR at $z=k$.

The improvement involved in this paper is toward using the magnetic field equations without any modifications and approximations:

$$
\left.H_{z}\right|_{i, j, k} ^{n+1 / 2}=\left.H_{z}\right|_{i, j, k} ^{n-1 / 2}+\frac{\Delta t}{\mu} \cdot\left\{\frac{\left.E_{x}\right|_{i, j, k} ^{n}-\left.E_{x}\right|_{i, j-1, k} ^{n}}{d y}-\frac{\left.E_{y}\right|_{i, j, k} ^{n}-\left.E_{y}\right|_{i-1, j, k} ^{n}}{d x}\right\}
$$

Similar equations can also be written for other magnetic-field components Fig. 1 shows the cross section of a cylindrical dielectric resonator along the z-axis, where, for convenience, we display only one of its quadrants. A regular cell that is completely filled with a homogenous medium, e.g., cell $(1,1, k)$, can be modeled by using the previous mentioned equations. However, these equations must be modified when a cell contains two different dielectric media, e.g., cell $(4,3, k)$, for which it is introduced the concept of an effective dielectric constant $\varepsilon_{\text {eff, }}$ which can be calculated by taking the weighted volume average of the two dielectrics and then expressed as:

$$
\varepsilon_{\text {reff }}=v_{s}(i, j, k) * \varepsilon_{r 1}+\left(1-v_{s}(i, j, k)\right) * \varepsilon_{r 2}
$$

where $v_{s}(i, j, k)$ is the fractional volume of the $(i, j, k)^{\text {th }}$ cell that contains the dielectric $\varepsilon_{r 1}$. $\varepsilon_{r 2}$ is the relative dielectric constant of the medium in the remaining volume. The procedure outlined above can be extended to cells containing more than two different dielectric media. Once the effective dielectric constants of the cells have been computed, the conventional FDTD method can be used to update the electric-field components.

\section{NUMERICAL RESULTS}

In order to demonstrate the propriety of the new FDTD method, some numerical results are presented. Fig. 2 illustrates the resonance frequencies of the cylindrical dielectric resonator shown in Fig. 3. Scattering parameters are calculated using fast Fourier transform (FFT) of the time-domain signal. The space steps are $0.36 \mathrm{~mm}$ in $\mathrm{x}$ - and $\mathrm{y}$-direction, and $0.3575 \mathrm{~mm}$ in z-direction. The computational regions are 
$16.56 \times 16.56 \times 10.725 \mathrm{~mm}^{3}$ meshed by $46 \times 46 \times 30$ cell $^{3}$. The relative permittivity of the dielectric cylinder is 22 , its radius is $5.21 \mathrm{~mm}$, and its height is $3.575 \mathrm{~mm}$, the length of the slot is $4 \mathrm{~mm}$, and its width is $1.08 \mathrm{~mm}$, the ground was considered of infinite dimensions. The structure was excited by a Gaussian pulse along the slot shown in Fig. 3, using perfectly matched layers (PML) the fields decay quickly at the distance 5-space steps from the outer surface of the dielectric resonator. The analysis was carried out for 4096 time steps and no instability was ever observed. Fig. 4 and Fig. 5 show the input impedance of the cylindrical dielectric resonator (CDR) calculated by the improved method and staircase mesh, respectively.

The percentage differences in the resonance frequencies are calculated for the adapted method and staircase FDTD grid relative to the measured resonance frequency $(6.41 \mathrm{GHz})[9]$. The results are compared with those derived by using the modal expansion technique given by Mittra [8]. The difference between the improved method and Mittra results are listed in Table I. It is clear that the improvement in the present method is better than in Mittra method.

Table I. Percentage improvement over staircase in the resonant frequency of cylindrical dielectric resonator computed by Mittra and present algorithms.

\begin{tabular}{|c|c|c|c|}
\hline \multirow{2}{*}{ Mode } & \multirow{2}{*}{ Mesh Size } & \multicolumn{2}{|c|}{$\begin{array}{c}\text { Percentage improvement over } \\
\text { staircase grids }\end{array}$} \\
\cline { 3 - 4 } & & Present & Mittra \\
\hline HEM $_{111}$ & $\lambda / 27$ & $49.22 \%$ & $22.72 \%$ \\
\hline
\end{tabular}

The resonant frequency for different resonator dimensions are illustrate in fig. 6 . In Fig. $6 a$ the scattering pararneter $S_{11}$ is calculated for radius is $5.21 \mathrm{~mm}$ and different heights of values $3.57,7.15$, and $10.45 \mathrm{~mm}$. In fig. $6 \mathrm{~b}$ the height is $3.47 \mathrm{~mm}$ and the radius has three values $5.22,7.98$, and $7.6 \mathrm{~mm}$. One can notice from the results that there is proportionality between the resonant frequencies and the ratio of the relative cavity volumes. Increasing the volume of the cavity decreasing the resonance frequency. Fig. 7 and Fig. 8 depicts the input impedance for the case illustrated in fig. 6 .

\section{CONCLUSIONS}

This paper has introduced a simple improvement of the conformal finite-difference time-domain scheme for accurately handling objects with curved dielectric bodies. The resonance frequencies of the cylindrical dielectric resonator are calculated by using this technique and the results show the improvement in the accuracy over the conformal FDTD scheme. 


\section{REFERENCES}

[1] K S Yee, "Numerical solution of initial boundary value problems involving Maxwell's equations in isotropic media," IEEE Trans. Antennas Propagat., vol. AP-14, pp. 302-307, May 1966.

[2] G. Mur, "Absorbing boundary conditions for the finite-difference approximation of the time-domain electromagnetic-fieldequations," IEEE Trans. Electromagn. Compat, vol. EMC-22, pp. 377-382, Nov. 1981.

[3] J. p. Berenger., "Perfectly matched layer for the FDTD solution of wavestructure interaction problems," IEEE Trans. Antennas Propagat., vol. 44, pp. 110-117, Jan. 1996.

[4] A. Mitkees, A. Allam, H. Hamed and O. Ali, "Time-Domain Analysis of Microstrip Line using FDTD," Al-Azhar University Engineering Journal, Vol. 5, pp. 293-302, Sep. 2000

[5] T. G. Jurgens and A. Taflove, K. Umashankar, and T, G. Moore, "Finitedifference time-domain modeling of curved surfaces," IEEE Trans. Antennas Propagat., vol. 40, pp. 357-366, Apr. 1992.

[6] R. Holland, "A finite difference time-domain EMP code in 3-D spherical coordinates," IEEE Trans. Nucl. Sci., vol. NS-30, pp.4592-4595, 1983.

[7] P. H. Harms, J. F. Lee, and R. Mittra, "A study of the nonorthogonal FDTD method versus the conventional FDTD technique for computing resonant frequencies of cylindrical cavities," IEEE Trans. Microwave Theory Tech., vol. 40, pp. 741-746, Apr. 1992

[8] Supriyo Dey and Raj Mittra, "A conformal finite-difference time-domain technique for modeling cylindrical dielectric resonator," IEEE Trans. Microwave Theory Tech., vol. 47, pp. 1737-1739, Sep. 1999

[9] Ahmad A. Kishk, A. Ittipiboon, Y. M. M. Anter, and M. Cuhaci, "Slot Excitation of the Dielectric Disk Radiator," IEEE Trans. Antennas Propagat., vol. 43, No. 2, pp. 198-201, Feb. 1995. 


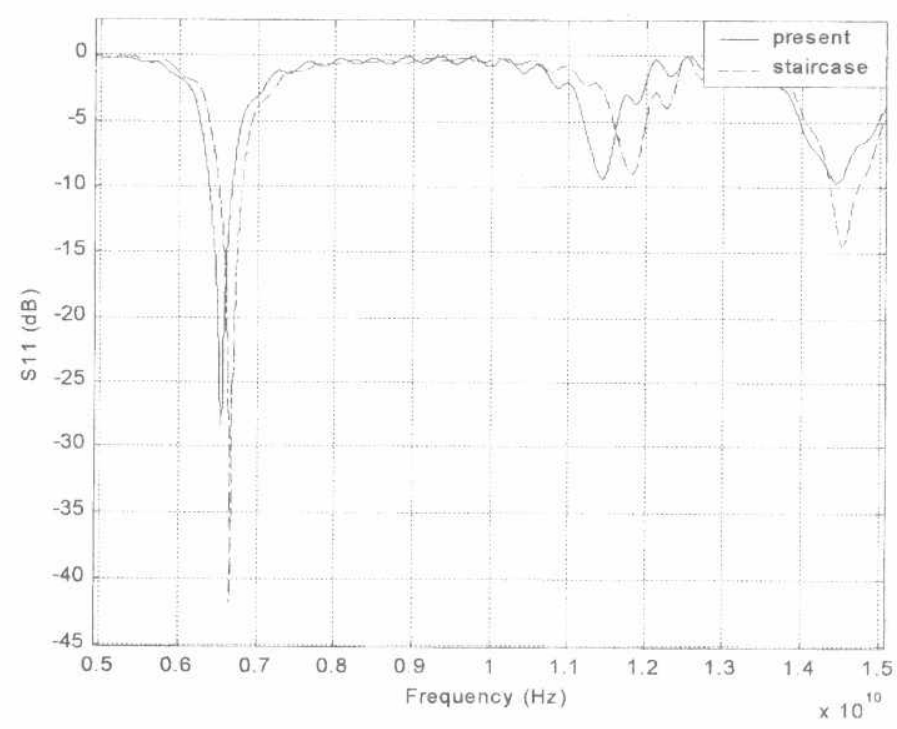

Fig. 2. S-parameters of cylindrical dielectric resonator.

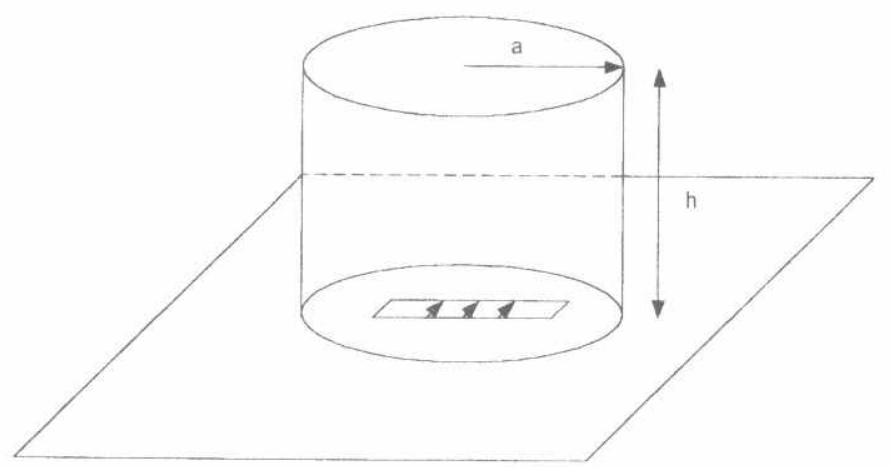

Fig. 3. Cylindrical dielectric resonator 


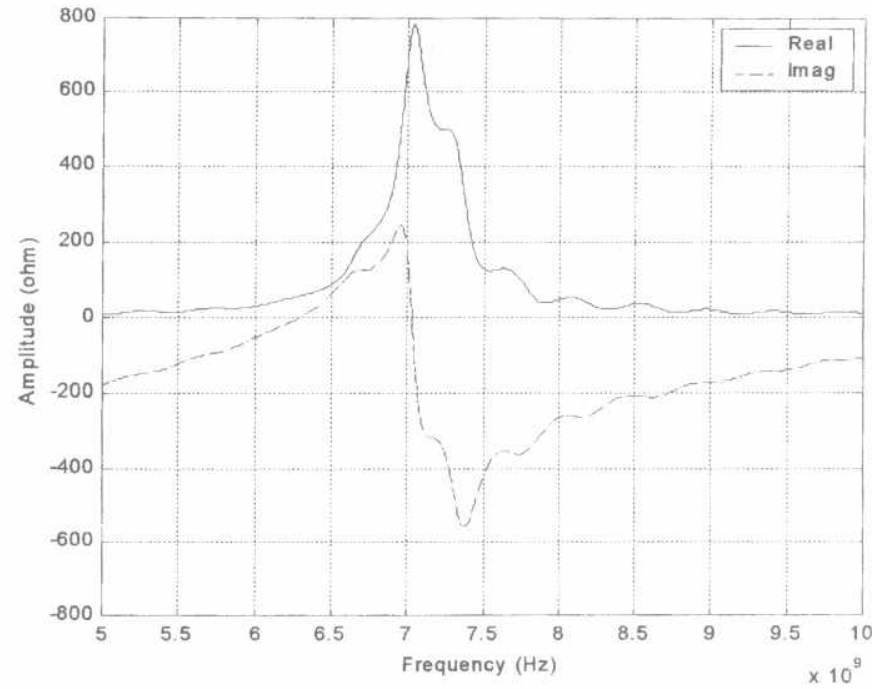

Fig. 4 Input impedance calculated by present method.

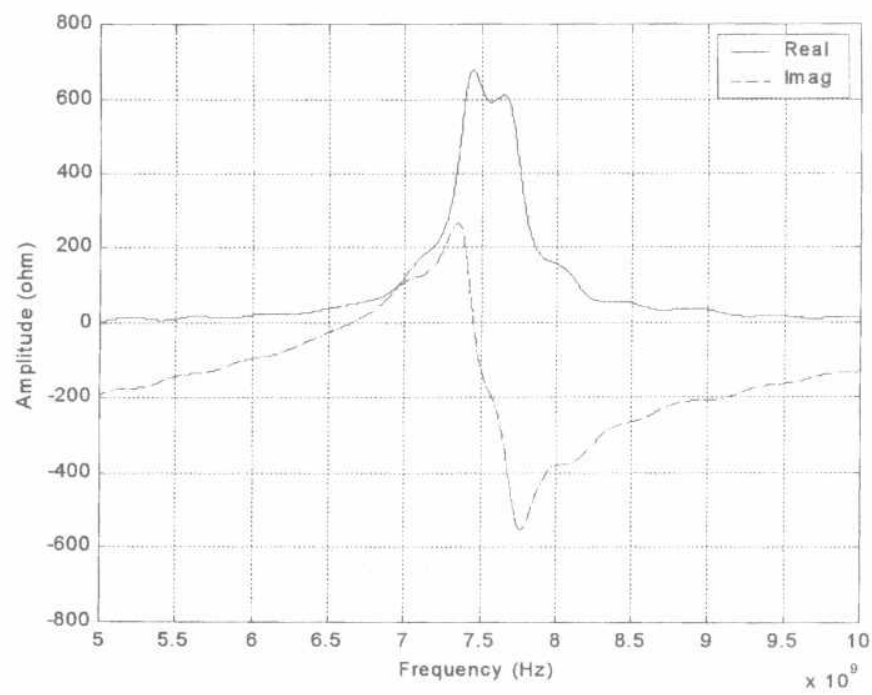

Fig. 5 Input impedance calculated by staircase method. 


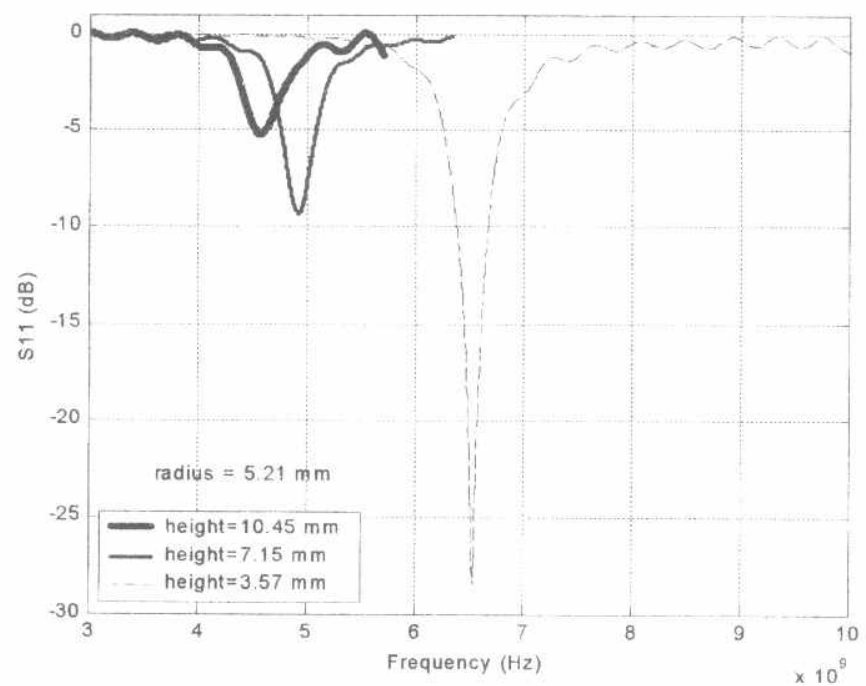

Fig.6a S-parameters of cylindrical dielectric resonator for different heights.

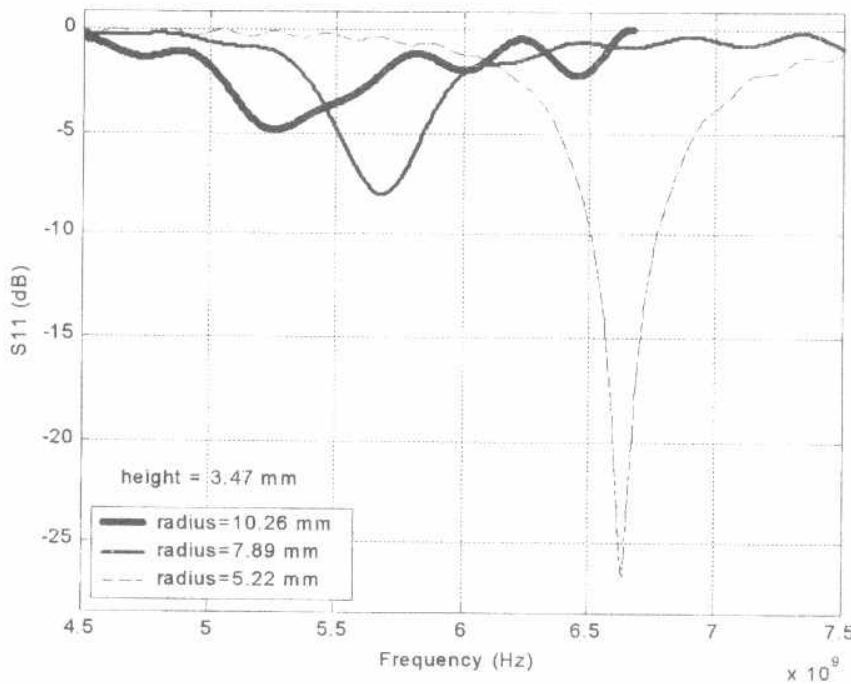

Fig.6b S-parameters of cylindrical dielectric resonator for different radius. 
Proceedings of the $9^{\text {th }}$ ASAT Conference, 8-10 May 2001

Paper BT -05

889

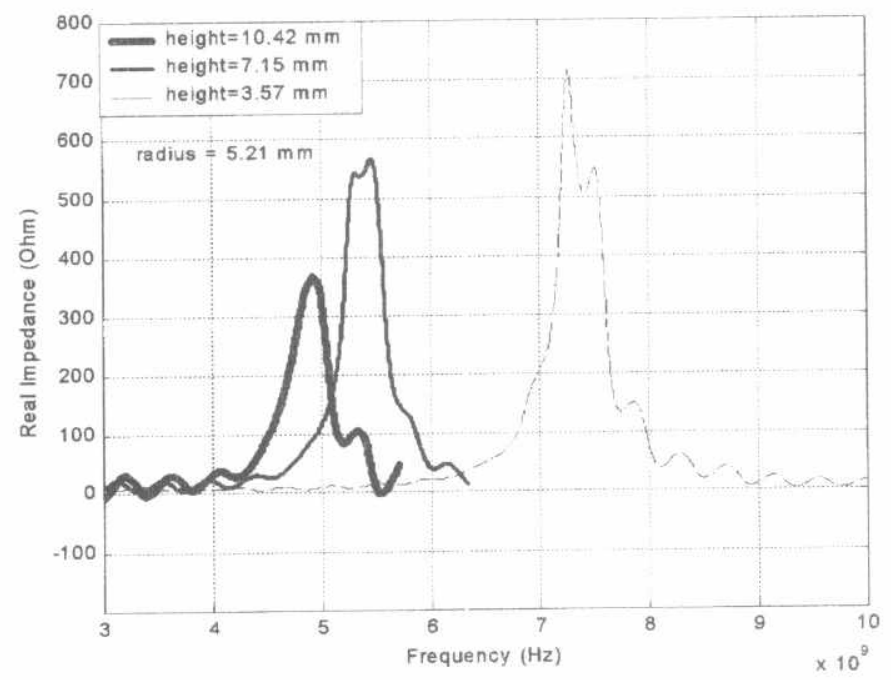

Fig.7a Real impedance of cylindrical dielectric resonator for different heights.

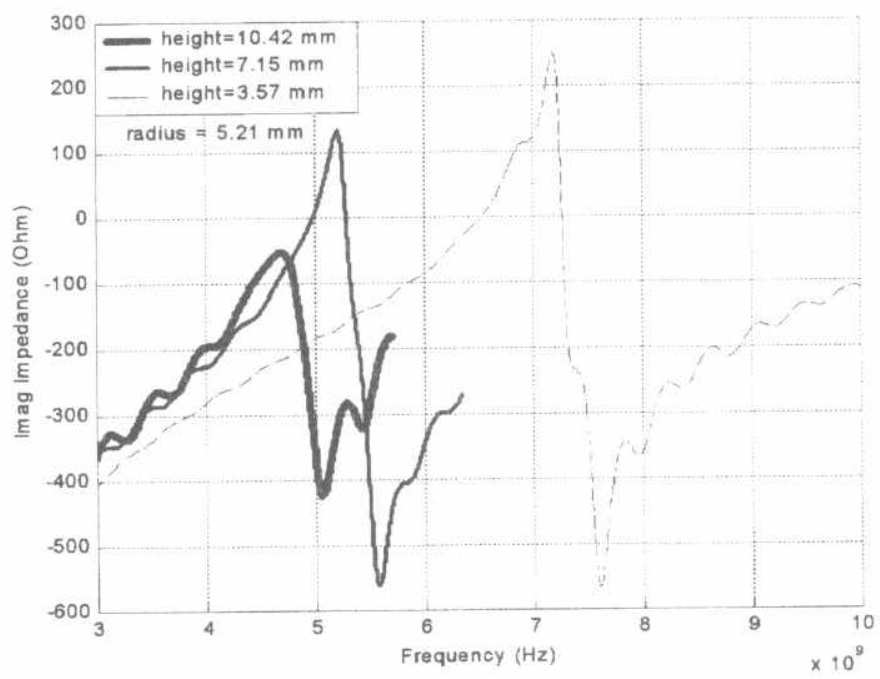

Fig. $7 \mathrm{~b}$ Imaginary impedance of cylindrical dielectric resonator for different heights. 


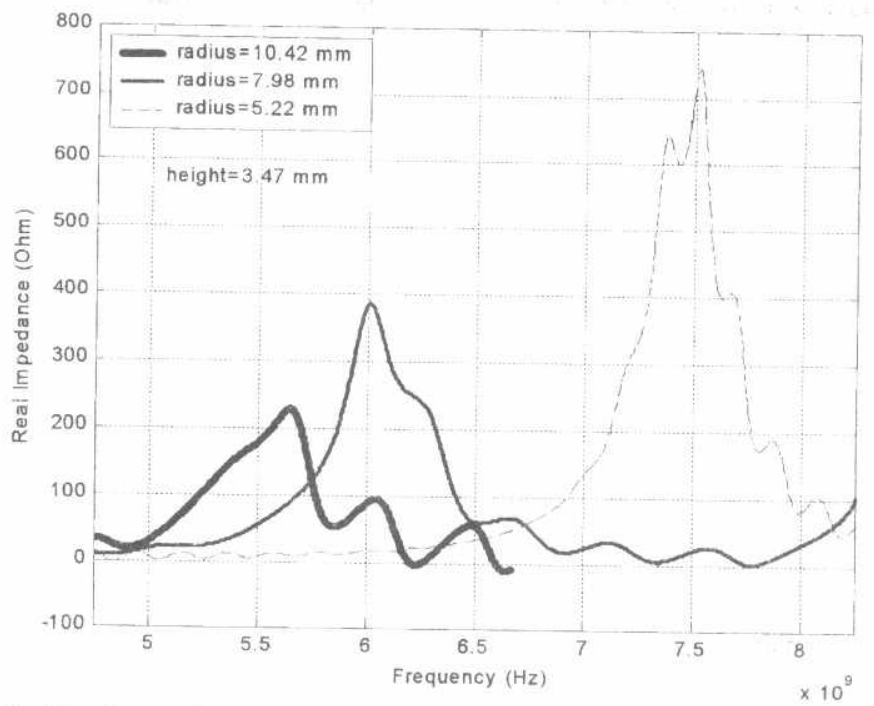

Fig. 8a Real impedance of cylindrical dielectric resonator for different radius

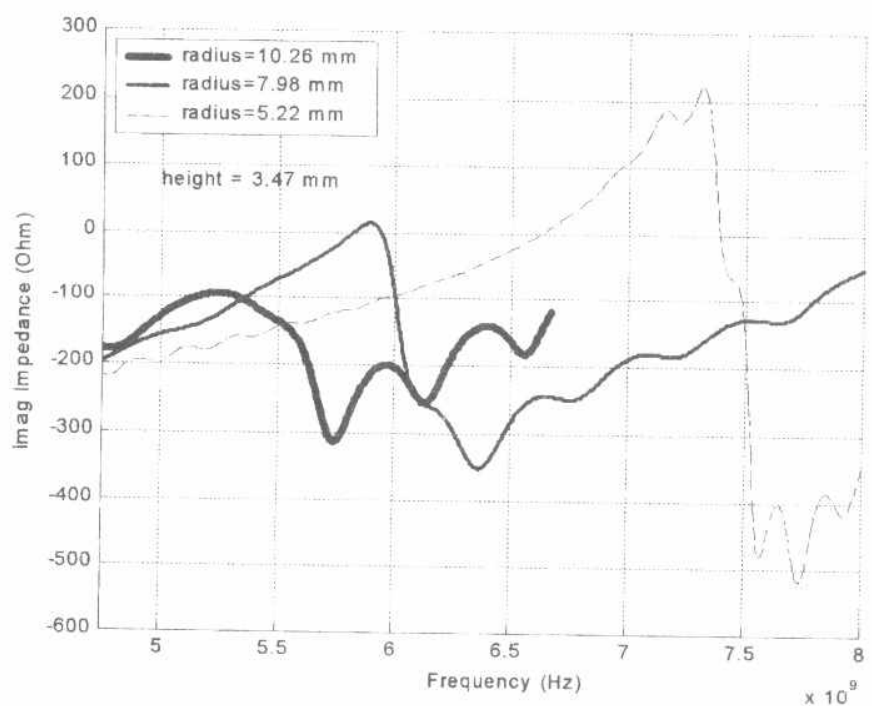

Fig. $8 \mathrm{~b}$ Imaginary impedance of cylindrical dielectric resonator for different radius. 